\title{
Recovery, Interrupted: The Zeigarnik Effect in EMDR Therapy and the Adaptive Information Processing Model
}

\author{
Jeremy G. Fox \\ Atlanta Georgia
}

\begin{abstract}
This theoretical article proposes that the Zeigarnik effect (ZE) (i.e., better memory for interrupted rather than completed tasks) explains the formation of traumatic memories as incomplete tasks. These tasks are thought to foster pathological rumination toward their completion while simultaneously stoking avoidance. After looking at the role of the ZE in the development and maintenance of posttraumatic stress disorder (PTSD), the article examines the physiological substrates of the ZE, including brain network imbalance, excessive autonomic nervous system activation, and prospective memory acuity for incomplete events. Eye movement desensitization and reprocessing (EMDR) therapy is proposed as uniquely capable of providing closure to interrupted facets of traumatic recollection, as this treatment may facilitate greater memory reconsolidation and inhibition of Zeigarnik reminders than extinction-based models. The ZE may be implicated in the overall EMDR treatment effect, as it is activated in several EMDR phases. Specifically, the use of brief interrupted exposures during EMDR reprocessing may benefit from the ZE in resolving traumatic events. Eye movements themselves are also considered interruptions to rumination upon traumatic memory. Recommendations for the further use of the ZE are described, and suggestions are made for future research. Protocol modifications and interweaves which mobilize the preceding insights are also provided.
\end{abstract}

Keywords: eye movement desensitization and reprocessing (EMDR) therapy; Zeigarnik effect; interoceptive accuracy (IAcc); feeling-of-knowing (FOK); Default Mode Network (DMN)

$\mathbf{T}$ he Zeigarnik effect (ZE) is a property of memory discovered in 1927 by eponymous psychologist Bluma Zeigarnik, who proposed that "interrupted tasks are better remembered than completed ones" (Seifert \& Patalano, 1991, p. 114). Her research shows that interruption of a task results in increased likelihood of recalling the task, and replications have confirmed her findings (Popova, 2015). It is now well known that "individuals tend to dwell and linger more on tasks that feel unfinished" (Pietrusza \& Hook, 2016, p. 107).

This theoretical article summarizes the findings from a large body of research on the ZE and examines how the $\mathrm{ZE}$ is implicated in the development and maintenance of posttraumatic stress disorder (PTSD). It examines the contribution of the ZE to several theories and summarizes possible related neurobiological mechanisms. Eye movement desensitization and reprocessing (EMDR) therapy is an efficacious treatment for PTSD. Several of its treatment components contain ZE-related mechanisms that probably contribute to EMDR's unique effectiveness and efficiency. The article explores the treatment implications and makes suggestions for how EMDR treatment might be enhanced by attending to the ZE.

\section{Introduction to the ZE}

The ZE entails intrusive memories and the binding of cognitive resources, both of which direct attention 
to the unfinished goal (Lewin, 1939; Masicampo \& Baumeister, 2011; Smallwood \& Schooler, 2006; Peifer, Syrek, Ostwald, Schuh, \& Antoni, 2019; Weigelt $\&$ Syrek, 2017). The focus on unfinished tasks, which occupies cognitive resources (Stocker et al., 2019; Syrek, Weigelt, Peifer, \& Antoni, 2017), has both positive and negative implications. On one hand, the $Z E$ is implicated in better memory, resolving impasses in problem solving, and insight-based learning (Sio $\&$ Ormerod, 2009). On the other hand, it is invoked to explain intrusive anger, cue-generated intrusive imagery that elicits past traumatic memories, and the drive to express anger after experiencing a perceived slight (Kühn et al., 2018; Liang et al., 2018; McHugh, Forbes, Bates, Hopwood, \& Creamer, 2012).

Seifert and Patalano (1991) posit that there is a tension system responsible for memorability, which directs attention, thoughts, perceptions, and attitudes toward task completion, and research has shown that active goals enhance accessibility of goal-related memories (Bongers, Dijksterhuis, \& Spears, 2010; Masicampo \& Baumeister, 2011). Incomplete work-related tasks correlate with higher arousal and difficulty in detaching from work (Smit, 2016). Task interruptions cause annoyance, anxiety, and feelings of stress (Bailey, Konstan, \& Carlis, 2001; Mark, Gudith, \& Klocke, 2008; Mark, Voida, \& Cardello, 2012). Words associated with an absence of closure are negatively associated with emotional inhibition, suggesting that psychological closure "may be a prerequisite for strategic emotional inhibition" (Beike, Adams, \& WirthBeaumont, 2007, p. 375).

The ZE is the motivational component of rumination, generated by failure to complete personally meaningful tasks (Martin \& Teeser, 1989). The ZE is therefore a memory bias toward completion of interrupted behavior. The interruption of a higher order goal causes subsequent intrusions (Gold \& Wegner, 1995).

\section{The ZE and the AIP Model}

EMDR therapy is based on Shapiro's (2001) Adaptive Information Processing (AIP) model, which describes an innate neurobiological mechanism which consolidates new memories into pre-existing networks. In current neuroscientific terminology, the information processing system asserted by the AIP model may be said to facilitate reconsolidation of traumatic memories into the appropriate past tense (Markus $\&$ Hornsveld, 2017). Shapiro proposed an innate information processing system that processes new experiences, producing an adaptive resolution "in which connections to appropriate associations are made and the experience is integrated into a positive emotional and cognitive schema" (Shapiro, 2017, p. 26). Disturbing experiences prevent the effective function of this system, leaving memories in an unprocessed and incomplete state. Pathological reliving or avoidance symptoms occur when these memories are triggered. From an AIP perspective, the ZE is related to the intrusive involuntary repetitions or ruminations arising from unprocessed and dysfunctionally stored traumatic memories.

\section{The ZE and Symptoms of PTSD}

The ZE is implicated in the development and maintenance of PTSD. ZE-related ruminations can be viewed as attempts to integrate traumatic experience into preexisting memory networks (Chemtob, Roitblat, Hamada, Carlson, \& Twentyman, 1988; Horowitz, 1974, 1976). Shapiro asserts that the "natural 'completion tendency' continues to rework the traumatic information in active memory until it can be reconciled with one's internal models of the world. Unless the trauma can be incorporated into existing schemata, the information will remain in active memory and will break through in intrusive thoughts. This process alternates with numbing and avoidance until some integration results" (Shapiro, 1995, p. 19).

Although rumination may facilitate integration, it may instead amplify subjective discomfort and result in overwhelm or subsequent avoidance, a paradoxical effect known as "Ironic Process" (McHugh et al., 2012, p. 97). Dual demands to both assimilate and avoid traumatic material are responsible for hyperarousal and avoidance characteristic of PTSD (Chemtob et al., 1988; Horowitz \& Becker, 1971), which thwart adaptive information processing (Shapiro, 2001). These "biphasic patterns" of "differentiation" (stimulus avoidance) and "linkage" (reliving and hyperarousal) (Laub, Nomi, \& Bender, 2017, pp. 115,116$)$ reflect the dualistic conceptualization of trauma dating back to Horowitz's work (Roşu, 2006). The $Z E$ can be understood to drive both "defense mechanisms," which excise traumatic material from consciousness, and "maintaining mechanisms," such as flashbacks and traumatic intrusions (James \& Kendell, 1997, p. 33). The ZE, or cognitive availability and cue sensitivity to events deemed incomplete, is synonymous with traumatic intrusions, which result in attempts to avoid cue stimuli, and which inhibit adaptive processing of traumatic information (James \& Kendell, 1997). 


\section{Interruptions and Perseveration About Safety}

Emotional discrepancy theory states that "emotions arise when schemas are disrupted and/or when current conditions conflict with established expectations or beliefs" (Harber \& Pennebaker, 1992, p. 362; Higgins, 1987; Mandler, 1964). Predictive Processing, a corollary to Karl Friston's Free Energy Principle, asserts that the brain actively predicts (infers) the current state of the external and internal environment to reduce free energy (entropy) (Chamberlin, 2019b). Any errors (surprises) in prediction are noticed, and either cognition or behavior are engaged, to realign reality with prediction. The surprise of prediction error may be the mechanism which initiates memory consolidation (Sinclair \& Barense, 2018). Encounters with a familiar stimulus, such as a movie segment, which feature an unanticipated interruption (i.e., a different ending to the film clip) register as surprising and initiate the process of memory reconsolidation (Sinclair \& Barense, 2018). This updates future inferences and expectations regarding the stimulus.

In this context, the ZE is evolutionarily adaptive, as it results in perseveration about safety after an interruption in goal-directed behavior. Dangerous experiences are encoded as more relevant to future predictions regarding safety and environmental cues which serve as reminders of the life-threatening event are afforded greater precision weighting (relevance) when subsequently encountered (Chamberlin, 2019b; Harber \& Pennebaker, 1992). Even if statistically unlikely, predictive coding gives greater weight to life-threatening predictions, and thus bases current actions and beliefs upon the presumption that threatening stimuli are still likely. Interrupted expectations are a crucial element in forging predictions for the future.

\section{Incompleted Physiological Responses}

Levine views traumatic intrusions as "incompleted" instinctive physiological responses, compelled by the autonomic nervous system (ANS) and musculature (Levine \& Frederick, 1997, p. 32). The initial ANS arousal is seen as a drive to resume interrupted tasks related to personal safety (fleeing) or acquisition of resources. This is an example of the $\mathrm{ZE}$ as an initially adaptive psychological phenomenon. The subsequent reactivation and post-event arousal compose the physiological basis of the ZE drive to ruminate, which impedes adaptive processing by focusing upon state-dependent fragments of memory (Gold \& Wegner, 1995). Thwarted physical reactions (i.e., inability to fight or flee from danger) are the basis of trauma itself, and result in a continued sensitivity to cue stimuli which provoke both the urge to express the previously interrupted physical action and a desire to avoid situations which trigger such vivid traumatic memories (Levin \& Frederick, 1997).

\section{Interrupted Tasks}

Interruptions do not always enhance memory. The $Z E$ is reversed when subjects receive the feedback that an interruption signifies positive performance and that completion means failure. In this case, completed tasks are remembered better than those deemed incomplete (Marrow, 1938).

Distractions can inactivate prospective memory intentions, as Einstein, McDaniel, Williford, Pagan, and Dismukes (2003) discovered, by testing memory for self-initiation of an incomplete task following interruption. However, remembering to restart a task, with no reminder provided, cannot be compared with the desire to resume an interrupted, inprogress task, as the latter entails emotional, subjectively relevant engagement. Reminders to complete interrupted tasks (of which the Einstein et al. (2003) study had none) effectively prime the ZE (Liberman, Forster, \& Higgins, 2007). Relatedly, it is much less likely for individuals suffering PTSD to forget the urge to complete trauma than it is for them to perseverate upon it or experience intrusive thoughts and cue reminders (Masicampo \& Baumeister, 2011). Furthermore, the ZE occurs in response to the interruption of actions with vital subjective significance, some of which are difficult and even unethical (in the case of trauma) to replicate in laboratory settings (Damir \& Toader, 2014).

\section{Somatic Experiencing Theory}

PTSD symptoms are forms of the ZE, as interrupted efforts at escaping or responding to threatening stimuli produce subsequent intrusions in the brain's information processing system (James \& Kendall, 1997). According to Somatic Experiencing Theory, stressful situations produce "survival actions" which, if interrupted, result in "vivid procedural memories" that recur as traumatic intrusions (Payne, Levine, \& CraneGodreau, 2015, p. 12). Ruminations serve to urge completion of "unresolved issues" and act in service of a goal-directed "completion tendency," which mobilizes the ANS response as well as mental predictions relevant to the previous traumatic event (James \& Kendall, 1997, p. 331; Levine \& Frederick, 1997 ). 
Familiar environmental cues may activate a feelingof-knowing which diverts attention from effortful, conscious activity and toward interrupted implicit (somatic) or psychological reactions (James \& Kendall, 1997; Payne et al., 2015). Feeling-of-knowing is "the awareness of an intention without remembering its content" (James \& Kendall, 1997, p. 330), and the sensation that information is available, despite unsuccessful attempts at retrieving it (Fiacconi, Kouptsova, \& Köhler, 2017). Feelings-of-knowing thus explain the avoidance or pursuit of environments evocative of threat or unfinished business, without explicit semantic or episodic memory awareness. Zeigarnik reminders (i.e., triggers which remind of incomplete resolution) are increased by the sensitivity to traumatic memory cues and subsequent conscious weighting (via feeling-of-knowing) of these triggers as relevant to the present moment (Chamberlin, 2019b). The ZE describes the persistence of predictions which pertain to past, easily remembered, and incomplete situations that are no longer relevant to the present. The ZE establishes an errant trajectory for prediction, which results in hypervigilant behavior and impedance of the AIP system. The ZE is conceptualized as a memory bias which urges traumatic rumination, and is known as a "drive for completion" (Horowitz \& Becker, 1971), "energetics" (Beck, 1996, p. 2), and "an energy force" which maintains "unprocessed information at an active subthreshold level" (James \& Kendall, 1997, p. 335) but routinely increases to the point of conscious awareness, via environmental cues which activate feelings-ofknowing.

\section{The ZE and EMDR Treatment Implications}

The role of the ZE is apparent in several EMDR treatment components. Related research provides insight into EMDR mechanisms. EMDR permits completion of Zeigarnik reminders across a range of past circumstances, via the multisensory nature of the assessment phase of the protocol and the apparent utility of eye movements to retrieve and reconsolidate episodic memories (Chamberlin, 2019b).

\section{Indirect Support for the AIP Model in ZE Research}

Some ZE research provides indirect support for Shapiro's (2017) AIP model. Findings show that interruption of an ongoing task improves subsequent recall of task elements, making the ZE a property of memory (Mantyla \& Sgaramella, 1997) and demonstrating how the ZE explains the stuck quality of unfinished traumatic tasks. When a task is completed, "goal-related constructs" are less accessible, and recall of the completed goal is inhibited (Liberman et al., 2007). These effects are predicted by the AIP model, which conceptualizes task completion as metabolization of episodic recollections into semantic memory (Shapiro, 2001). With completion of the task and achievement of the goal, the ZE and its maintaining mechanisms are extinguished (Liberman et al., 2007). The AIP model asserts that desensitization and reprocessing are the "byproducts of adaptive reprocessing ... on a neurophysiological level" (Shapiro, 1995, p. 13).

Shapiro (2001) posited that "interrupted exposure," consisting of short bilateral stimulation (BLS) sets alternated with "cognitive debriefing," appears to be a crucial element of EMDR reprocessing (p. 318). EMDR functions via continued titration between memory exposure and metacommunication (Boudewyns \& Hyer, 1996). The "distancing process" (i.e., a focus upon material other than the traumatic memory, such as eye movements and therapist verbal feedback) is associated with greater symptom improvement than exposure alone (Lee, Taylor, \& Drummond, 2006, p. 105). There is thus a precedent for positing interruption as an innate mechanism of EMDR treatment effectiveness.

\section{Importance of ZE During Activation of the Memory Components in the Assessment Phase}

EMDR processing sessions begin with the assessment phase, during which clients recall sensory, cognitive, affective, and somatic aspects of the target memory. Although most EMDR procedures have inherent flexibility, the assessment procedure is quite scripted, as Shapiro (1995, p. 135) thought it was essential to activate all components of the memory network.

The ZE adds another element to understanding the importance of this practice. This article proposes that activation of the memory network initiates the ZE drive for completion, which presses for elicitation of the traumatic, somatic memory as well as maintaining mechanisms, which attempt to sustain traumatic memory. Impulses toward resolving incomplete actions are also activated, thus strengthening motivation to work on resolution in the next phases of the treatment process. Additionally, in the EMDR assessment phase, the client articulates a desired selfreferencing cognition, which does not yet feel valid. 
This further activates the drive for completion, by interrupting the ruminative focus upon the task of reprocessing the target memory. An urge toward actualizing the future positive goal as an interrupted task is also potentially initiated.

Prospective memory is "the ability to remember to perform an intended action at a given time in the future" (Mioni, Santon, Stablum, \& Cornoldi, 2017, p. 588). It entails two elements: recollection of memory at the appropriate time (the prospective aspect), and remembrance of the task itself (the retrospective component) (Einstein \& McDaniel, 1990, 1996; Kliegel, McDaniel, \& Einstein, 2008). Clients often recognize that a traumatic event is incomplete (the retrospective component) but fail to actualize prospective intent. EMDR may alleviate both the retrospective facet of prospective memory (via the assessment phase of the standard protocol) and the prospective element (through desensitization, reprocessing, installation, and future templates).

\section{ZE During Interruptions of Dual Attention Task During EMDR Processing}

During EMDR processing, the client is repeatedly interrupted by "check-ins" while focusing on the memory. This interrupting stimulus is likely to heighten the ZE and motivation to complete processing (Chamberlin, 2019a; Levine \& Frederick, 1997, p. 32). The interruption of a task catalyzes better prospective memory performance upon later attempts at solving the task (Mantyla \& Sgaramella, 1997). Interruption is hypothesized to endow the memory of the unfinished task with salience or "activation," which sustains attention toward conclusion of the action, and increases readiness to recognize environmental cues that remind of the goal's incompletion (p. 195). These "Zeigarnik-like effects" of memory (p. 192) suggest that the drive to complete active intentions is increased by further interruptions.

The EMDR treatment process is comprised of multiple brief periods (e.g., about 30 seconds) of dual attention tasks, during which the client focuses on aspects of the traumatic memory while simultaneously engaging in BLS. BLS in the form of eye movements is considered interrupting "white noise," which is proposed to restore thalamo-cortical activity, allowing a return to adaptive processing of memory (Miller, McGowan, Bergmann, Farrell, \& McLaughlin, 2018, p. 218). This, alongside interruptions ("check-ins"), activates the $\mathrm{ZE}$ as a drive to integrate and inhibit traumatic memories. The task of reprocessing is interrupted to elicit associations, after which the client again resumes the task with subsequent frequent repeated interruptions. During the dual attention tasks, the EMDR client experiences brief exposures to the traumatic memory. This interrupted exposure differs greatly from that used in cognitive behavioral therapies such as prolonged exposure, in which clients are asked to describe the memory as vividly as possible for about 60 minutes during a 90-minute session. The therapist interrupts the process about every five minutes to ask for more details or for levels of subjective distress (Foa, Hembree, \& Rothbaum, 2007). Although a large body of empirical evidence shows that lengthy exposure is not needed for EMDR's efficacy, until now there has never been any indication about why short exposures might be effective. However, it is now apparent that the ZE may play a critical role.

\section{Heart Rate, Interoception, and Feelings-of- Knowing as Substrata of the ZE}

Interoception is described as the body's internal milieu (Barrett, 2017), reminiscent of the "inner model" proposed by Horowitz (1974, 1976). Interoception is the "core of the brain's internal model" (Barrett, 2017, p. 6), further defined as the "afferent signaling of the internal state of the body" (Critchley \& Garfinkel, 2018 , p. 13), "the ability to perceive internal bodily signals" (Fiacconi et al., 2017, p. 71), and "the representation and utilization of ... internal resources" (Barrett, 2017, p. 6). Interoception is also cited as a "basis for subjective feelings and emotions" (Calì, Ambrosini, Picconi, Mehling, \& Committeri, 2015, p. 1) because "Subjective emotional experience is shaped by bodily conditions" (De Witte, Sütterlin, Braet, \& Mueller, 2016, p. 3). This supports the notion that emotional states and cognitive processes originate from physiological activity.

Interoceptive accuracy is defined as the "performance on objective behavioral tests of heartbeat detection" (Garfinkel, Seth, Barrett, Suzuki, \& Critchley, 2015, p. 65). The constituent elements of interoception are the subjects of recent refinement, as interceptive awareness is no longer considered synonymous with interoceptive accuracy. All instances of interoceptive accuracy in the present article refer to "performance on objective behavioral tests of heartbeat detection."

Interoceptive accuracy is significant to the ZE. Interoceptive accuracy is reported to improve memory (Garfinkel et al., 2013) and decision-making (Werner et al., 2013), and is a mediating factor between heart rate and prospective memory. 
Increased heart rate improves prospective memory retrieval (Umeda, Tochizawa, Shibata, \& Terasawa, 2016). Poor interoceptive accuracy is implicated in a host of psychopathologies, such as dissociative disorders (Schäflein, Sattel, Pollatos, \& Sack, 2018), and both anxiety-related and depression-related distress (especially when high levels of rumination are coupled with low levels of interoceptive accuracy) (Lackner \& Fresco, 2016). As prospective memory is susceptible to Zeigarnik-like effects, prior traumatic experiences take the form of prospective memory tasks. These traumatic memories are treated as targets for resolution through the EMDR protocol.

Following memory cue (stressor) presentation, psychological stress results in heart rate increase and memory retrieval (Schell \& Catania, 1975; Umeda et al., 2016; Zeigarnik, 1927). This is understood to be the basis of the tension system responsible for the unpleasant urge to complete unfinished intentions (Seifert $\&$ Patalano, 1991). "According to the implications of the Zeigarnik effect, individuals tend to experience psychological stress when sustaining a delayed intention" (Umeda et al., 2016, p. 2). Readers will recall that rumination is considered synonymous with the $\mathrm{ZE}$ and is prompted by ANS activation (Gold \& Wegner, 1995).

Additionally, the utility of increased heart rate to memory retrieval is relevant to EMDR, as PTSD survivors often demonstrate elevated basal heart rates (Hopper, Spinazzola, Simpson, \& van der Kolk, 2006). Interoceptive accuracy may be indispensable to successful recall and diminished interoceptive accuracy may explain "fragmented" traumatic memory retrieval (Lanius \& Bergmann, 2014, p. 221). Furthermore, a feeling-of-knowing judgment precedes prospective memory retrieval (Koriat \& Levy-Sadot, 2001; Reder, 1987; Umeda et al., 2016). Feelings-ofknowing are epistemic feelings, positively correlated with increased heart rate in response to familiar cues, as mediated by interoceptive accuracy (Fiacconi et al., 2017, p. 71). This suggests that psychological stress increases heart rate, resulting in a feeling-of-knowing judgment that aids in prospective memory retrieval, as mediated by interoceptive accuracy (i.e., the ability to "tune in" to bodily, cardiac information (Fiacconi et al., 2017, p. 77). Emotionally salient triggers serve as prospective memory reminder cue stimuli, eliciting feelings-of-knowing, increased heart rate, and a search for relevant state-dependent memory, mediated by interoceptive accuracy (Mantyla \& Sgaramella, 1997; Umeda et al., 2016). These are physiological mechanisms of the ZE.
Cue memory sensitivity influences salience detection systems (i.e., the salience network), greater activation of autonomic reactivity, subsequent elicitation of feeling-of-knowing, and memory recall (Chamberlin, 2019a; Umeda et al., 2016; West, Jakubek, \& Wymbs, 2002). Imbalance between the salience network and the self-referential processing default mode network (DMN) is implicated in the reliving and avoidance symptoms entailed in PTSD (Chamberlin, 2019a, p. 134; Laub et al., 2017).

Recall of state-dependent memories can overwhelm the thalamus, impeding memory integration (Lanius \& Bergmann, 2014; Lanius, Paulsen, \& Corrigan, 2014). This may result in subsequent avoidance of environmental cues and suppression of interoceptive, salience network awareness (Aupperle et al., 2012; Chamberlin, 2019a). The DMN self-referential brain network may then produce maladaptive ruminations and thought intrusions which discount the present context (Fingelkurts \& Fingelkurts, 2019). This is deemed the "vicious cycle" of brain network imbalance activity, and further describes the physiological configuration of ZE dysfunction (Chamberlin, 2019a, p. 134).

\section{Utility of ZE to EMDR Interventions and Interweaves}

The following modifications are suggested, regarding the $\mathrm{ZE}$ as a treatment concern.

\section{Task Completion}

A driving force in therapy, task completion is subjective in nature and clients must feel satisfied with the conclusion of their interrupted tasks (Beike et al., 2007; McNamara \& Lister-Ford, 1995). This push for completion is intensified by the use of interruptions during EMDR processing. The following outlines several ways in which this can be accomplished.

Somatic Discharge and Somatic Interweaves. Levine's Somatic Experiencing therapy asserts that physical movement which was thwarted during the precipitating (traumatic) event must be reenacted in order to release the implicit memories responsible for reliving symptoms (Payne et al., 2015). Somatic discharge may be integral for stuck clients, as persons typically regret interrupted, prevented action (freezing; inaction) more than action (Savitsky, Medvec, \& Gilovich, 1997). Thus, somatic interweaves may 
enable resolution of traumatic experience. Especially significant are "somatic micromovements" for reprocessing "trapped sympathetic arousal" (Paulsen \& Lanius, 2009, p. 358).

Blocked Processing. A Zeigarnik view holds that clients unresponsive to EMDR are insufficiently catalyzed by the drive to completion, as it is thwarted by defense mechanisms. Therefore, incomplete scenarios may be resolved through direction of attention toward desired alternative responses during past traumatic events. Linkage with positive memory networks is already entailed in the EMDR installation phase; however, it may be necessary to emphasize the reenacting element of the protocol further, through interweaves which provide symbolic completion of events. Somatic interweaves may be effective due to the importance of reenacting blocked, unexpressed intentions, allowing for resolution of the ZE (James $\&$ Kendall, 1997). Blocked processing requires greater activation of memory elements. Fortunately, modifications for activating differing facets of memory are available; notable is the Bottom-Up Protocol, tasked with increasing somatic recall (Paulsen \& Lanius, 2009).

Future research may create and test a modified protocol that emphasizes the incomplete action urges or intentions thwarted by traumatic experience for those who underaccess traumatic memories and associated emotions. Direction from the EMDR clinician toward interrupted intentions or physiological reactions may activate rumination and bypass avoidance, triggering the emotions and interoceptive sense necessary for memory retrieval and effective EMDR reprocessing. There is precedent for such specific focus in the EMDR protocol, which restricts processing to only one memory (Hensley, 2016).

Such a proposed protocol for underaccessing emotion may explicitly draw upon the responsibility, safety, and choice plateaus that represent different cognitive orientations toward the traumatic memory (Hensley, 2016). Plateau-dependent reprocessing of thwarted safety, ruminations upon responsibility, or perseveration upon lack of control is extensively used as an interweave, but typically combined with a focus upon completion and used for those who overaccess the target memory. For clients who underaccess, explicit attention may be directed toward the above plateaus, with a focus upon their lack of resolution. The resulting attention upon interrupted safety, choice, or errant assumption of responsibility (i.e., "I should have done something different") may activate somatic memories of thwarted intentions, harnessing the ZE.

Session Closure. Therapy sessions concluded with impasses may direct attention to therapeutic goals (Pietrusza \& Hook, 2016). If incomplete sessions are closed with appropriate exercises, utilizing this urgency toward resolution of targets may prove beneficial. The ZE may be employed to increase drive for resolution between standard protocol sessions and prove helpful in highlighting traumatic fragments for integration.

\section{Target Selection}

Conceptualizing EMDR targets as Zeigarnik interruptions to the pursuit of higher order goals, such as relationship maintenance (as in the case of a loved one who has passed, a friend with whom communication has been halted, or a child who has moved from the home), may benefit treatment planning (Gold $\&$ Wegner, 1995). The expression of unspoken, interrupted statements toward such attachment figures may serve as valuable interweaves during reprocessing.

\section{The Future Template}

EMDR can even generalize the newfound psychological mastery established by such physiological actions into further situations, via the future template. A preemptive emphasis upon thwarted or interrupted coping may be adaptive within the future template phase of the three-pronged EMDR protocol and serve as a method for inoculation against further Zeigarnik reminders related to the same target issue. EMDR emphasizes the generalization of acquired insights and competencies (known as resources) through memory networks not only related to the past, but also to anxiety memory networks which pertain to the present and beyond. For instance, a client who struggles with anxious attachment may be instructed to imagine how he or she will respond to an online dating match disappearing or "ghosting" in the future, as a tangible example of interrupted relational goals. This focus upon the ZE allows for a richer exploration of the role subjective anxieties inhabit within client lives.

Additionally, installing future desired states as positive templates with open-ended focus may encourage striving toward accomplishing incomplete goals. For example, installing "I will engage in hikes for self-care" with imagery of beginning a hike may harness the power of the ZE toward adaptive ends. Interrupting 
the installation of the Positive cognition may encode the $\mathrm{PC}$ as a prospective memory intention, increasing susceptibility to elicitation by environmental cues that could remind the client to hike for a self-care break. This might sustain the drive to completion better than an uninterrupted adaptive goal.

\section{Conclusion}

It is apparent that a Zeigarnik-informed approach to EMDR may enhance the standard protocol. The preceding work provides evidence of the $\mathrm{ZE}$ as a heuristic that describes the hindrance of adaptive information processing and situates the ZE within the broader EMDR research base. It has been the purpose of this article to illuminate the elegance of the EMDR protocol in relieving pathological symptoms through completion of the psychological and physiological tension caused by traumatic events. The assessment phase of EMDR, eye movements, and brief interruptions (or "check-ins") were credited with eliciting the ZE, while eye movements were also theorized to initiate adaptive reprocessing of ruminative cognitions and autonomic arousal. Physiological underpinnings of the ZE were also hypothesized, including the role of cue reminders in increasing heart rate, resulting in feelings-of-knowing and subsequent memory retrieval, as mediated by interoceptive accuracy. Network brain imbalance was also examined as a source of the rumination that characterizes the ZE. Traumatic experiences were conceptualized as prospective memory tasks, the resolution of which EMDR is distinctively suited. Interweaves and protocol modifications were offered, which directly addressed the incomplete tasks and prospective memories formed by trauma.

Future research is recommended to further investigate the ZE within EMDR therapy. Experiments could examine the role of the interrupted dual task of BLS during EMDR processing by comparing standard EMDR procedures with a variant in which no interruptions are used and the client engages in lengthy exposures. The role of the ZE in the assessment phase could also be examined. Studies may experiment with assessment phase format, encouraging client focus upon incomplete elements of the traumatic memory in the "image" section of the protocol. An example could include replacement of the question, "What image represents the worst part of the memory," with "what image does your mind continue to replay?" or "What image or part of the memory do you wish had a different ending?” These protocol modifications might catalyze blocked or non-existent reprocessing in those who underaccess traumatic memory. Such clients are often beset by avoidance mechanisms.

Researchers could examine prospective memory task performance, feeling-of-knowing judgments, and interoceptive accuracy as correlated with EMDR treatment responsiveness, to test the proposed relevance of the above physiological constructs to both the ZE and EMDR treatment. Other enhanced ZE interweaves and modifications could also be investigated.

\section{References}

Aupperle, R. L., Allard, C. B., Grimes, E. M., Simmons, A. N., Flagan, T., Behrooznia, M., ... Stein, M.B. (2012). Dorsolateral prefrontal cortex activation during emotional anticipation and neuropsychological performance in posttraumatic stress disorder. Archives of General Psychiatry, 69(4), 360-371. https: / / doi.org/ 10.1001 / archgenpsychiatry.2011.1539

Bailey, B. P., Konstan, J. A., \& Carlis, J. V. (2001). The effects of interruptions on task performance, annoyance, and anxiety in the user interface. Interact, 1, 593-601.

Bongers, K. C., Dijksterhuis, A., \& Spears, R. (2010). On the role of consciousness in goal pursuit. Social Cognition, 28(2), 262-272.

Barrett, L. F. (2017). The theory of constructed emotion: An active inference account of interoception and categorization. Social Cognitive and Affective Neuroscience, 12(1), 1-23. https: / / doi.org/10.1093/scan/nsx060

Beck, A. T. (1996). Beyond belief: A theory of modes, personality and psychopathology. In P. M. Salkovskis (Ed.), Frontiers of cognitive therapy. Guilford.

Beike, D. R., Adams, L. P., \& Wirth-Beaumont, E. T. (2007). Incomplete inhibition of emotion in specific autobiographical memories. Memory, 15(4), 375-389. https: / / doi.org/10.1080/09658210701276850

Boudewyns, P. A., \& Hyer, L. A. (1996). Eye movement desensitization and reprocessing (EMDR) as treatment for post-traumatic stress disorder (PTSD). Clinical Psychology \& Psychotherapy: An International Journal of Theory and Practice, 3(3), 185-195.

Calì, G., Ambrosini, E., Picconi, L., Mehling, W. E., \& Committeri, G. (2015). Investigating the relationship between interoceptive accuracy, interoceptive awareness, and emotional susceptibility. Frontiers in psychology, 6, 1-13. https: / / doi.org/10.3389/fpsyg.2015.01202

Chamberlin, D. E. (2019a). The network balance model of trauma and resolution-level I: Large-scale neural networks. Journal of EMDR Practice and Research, 13(2), 124-142. https: / / doi.org/10.1891/1933-3196.13.2.124

Chamberlin, D. E. (2019b). The predictive processing model of EMDR. Frontiers in Psychology, 10, 1-14. https: / / doi.org/10.3389/ fpsyg.2019.02267 
Chemtob, C., Roitblat, H. L., Hamada, R. S., Carlson, J. G., \& Twentyman, C. T. (1988). A cognitive action theory of post-traumatic stress disorder. Journal of Anxiety Disorder, 2, 253-275. https:/ / doi.org/10.1016/ 0887-6185(88)90006-0

Critchley, H. D., \& Garfinkel, S. N. (2018). The influence of physiological signals on cognition. Current Opinion in Behavioral Sciences, 19, 13-18. https://doi. org/10.1016/j.cobeha.2017.08.014

Damir, D., \& Toader, E. (2014). Posttraumatic stress syndrome-ethical and biopsychosocial implications. Procedia-Social and Behavioral Sciences, 149, 276-279. https: / / doi.org/10.1016/j.sbspro.2014.08.229

De Witte, N. A., Sütterlin, S., Braet, C., \& Mueller, S. C. (2016). Getting to the heart of emotion regulation in youth: The role of interoceptive sensitivity, heart rate variability, and parental psychopathology. PLOS ONE, 11(10), p. 3 e0164615. https://doi.org/ 10.1371 / journal.pone. 0164615

Einstein, G. O., \& McDaniel, M. A. (1990). Normal aging and prospective memory. Journal of Experimental Psychology: Learning, Memory, and Cognition, 16(4), 717. https: / / doi.org/10.1037/0278-7393.16.4.717

Einstein, G. O., \& McDaniel, M. A. (1996). Retrieval processes in prospective memory: Theoretical approaches and some new empirical findings. In Brandimonte, M.A., Einstein, G. O., \& McDaniel, M.A. (Eds.), Prospective memory: Theory and applications (pp. 115-141). Psychology Press. https://doi.org/ $10.4324 / 9781315806488$

Einstein, G. O., McDaniel, M. A., Williford, C. L., Pagan, J. L., \& Dismukes, R. (2003). Forgetting of intentions in demanding situations is rapid. Journal of Experimental Psychology: Applied, 9(3), 147. https:// doi.org/10.1037/1076-898X.9.3.147

Ferri, F., Ardizzi, M., Ambrosecchia, M., \& Gallese, V. (2013). Closing the gap between the inside and the outside: Interoceptive sensitivity and social distances. PLOS ONE, 8(10), e75758. https:// doi.org/10.1371/journal.pone.0075758

Fiacconi, C. M., Kouptsova, J. E., \& Köhler, S. (2017). A role for visceral feedback and interoception in feelingsof-knowing. Consciousness and Cognition, 53, 70-80. https: / / doi.org/10.1016/j.concog.2017.06.001

Fingelkurts, A. A., \& Fingelkurts, A. A. (2019). Eye movement desensitization and reprocessing for post-traumatic stress disorder from the perspective of three-dimensional model of the experiential selfhood. Medical Hypotheses, 131, 109304. https: / / doi.org/10.1016/j.mehy.2019.109304

Foa, E., Hembree, E., \& Rothbaum, B. O. (2007). Prolonged exposure therapy for PTSD: Emotional processing of traumatic experiences therapist guide. Oxford University Press.

Garfinkel, S. N., Barrett, A. B., Minati, L., Dolan, R. J., Seth, A. K., \& Critchley, H. D. (2013). What the heart forgets: Cardiac timing influences memory for words and is modulated by metacognition and interoceptive sensitivity. Psychophysiology, 50(6), 505-512. https: / / doi.org/10.1111/psyp.12039

Garfinkel, S. N., Seth, A. K., Barrett, A. B., Suzuki, K., \& Critchley, H. D. (2015). Knowing your own heart: Distinguishing interoceptive accuracy from interoceptive awareness. Biological Psychology, 104, 65-74. https: / / doi.org/10.1016/j.biopsycho.2014.11.004

Gold, D. B., \& Wegner, D. M. (1995). Origins of ruminative thought: Trauma, incompleteness, nondisclosure, and suppression. Journal of Applied Social Psychology, 25(14), 1245-1261. https://doi.org/10.1111/j.15591816.1995.tb02617.x

Harber, K. D., \& Pennebaker, J. W. (1992). Overcoming traumatic memories. In S. Christianson (Ed.), The handbook of emotion and memory: Research and theory (pp. 359-387). Psychology Press. https://doi. org/10.4324/9781315807454

Hensley, B. J. (2016). An EMDR therapy primer: From practicum to practice (2nd ed.). Springer Publishing.

Higgins, E. T. (1987). Self-discrepancy: A theory relating self and affect. Psychological Review, 94(3), 319. https: / / doi.org/10.1037/0033-295X.94.3.319

Hopper, J. W., Spinazzola, J., Simpson, W. B., \& van der Kolk, B. A. (2006). Preliminary evidence of parasympathetic influence on basal heart rate in posttraumatic stress disorder. Journal of Psychosomatic Research, 60, 83-90. https:// doi.org/10.1016/j.jpsychores.2005. 06.002

Horowitz, M. (1974). Stress response syndromes: Character style and dynamic psychotherapy. Archives of General Psychiatry, 31(6), 768-781. https://doi.org/ 10.1001 / archpsyc.1974.01760180012002

Horowitz, M. J. (1976). Stress response syndromes. Jason Aronson.

Horowitz, M. J., \& Becker, S. S. (1971). Cognitive response to stressful stimuli. Archives of General Psychiatry, 25, 419-428. https://doi.org/10.1001/ archpsyc.1971.01750170035007

James, I. A., \& Kendell, K. (1997). Unfinished processing in the emotional disorders: The zeigarnik effect. Behavioural and Cognitive Psychotherapy, 25(4), 329-337. https: / / doi.org/10.1017/S1352465800018737

Kliegel, M., McDaniel, M. A., \& Einstein, G. O. (Eds.). (2008). Prospective memory: Cognitive, neuroscience, developmental, and applied perspectives. Taylor \& Francis.

Koriat, A., \& Levy-Sadot, R. (2001). The combined contributions of the cue-familiarity and accessibility heuristics to feelings of knowing. Journal of Experimental Psychology: Learning, Memory, and Cognition, 27(1), 34. https://doi.org/10.1037/0278-7393. 27.1 .34

Kühn, S., Düzel, S., Drewelies, J., Gerstorf, D., Lindenberger, U., \& Gallinat, J. (2018). Psychological and neural correlates of embitterment in old age. Psychological Trauma: Theory, Research, Practice, and Policy, 10(1), 51. https: / / doi.org/10.1037/ tra0000287 
Lackner, R. J., \& Fresco, D. M. (2016). Interaction effect of brooding rumination and interoceptive awareness on depression and anxiety symptoms. Behaviour Research and Therapy, 85, 43-52. https:// doi.org/10.1016/j.brat.2016.08.007

Lanius, U. (2009). The bottom-up protocol. In M. Luber (Ed.), Eye movement desensitization and reprocessing (EMDR) scripted protocols: Special populations (pp. 349356). Springer. https: / / doi.org/10.1891/978082612245

Lanius, U. F., \& Bergmann, U. (2014). Dissociation, EMDR, and adaptive information processing: The role of sensory stimulation and sensory awareness. In U. F. Lanius, S. L. Paulsen, \& F. M. Corrigan (Eds.), Neurobiology and treatment of traumatic dissociation: Toward an embodied self (pp. 213-233). Springer Publishing Company. http: / / doi.org/10.1891/9780826106322

Lanius, U. F., Paulsen, S. L., \& Corrigan, F. M. (2014). Dissociation: Cortical deafferentation and the loss of self. In U. F. Lanius, S. L. Paulsen, \& F. M. Corrigan (Eds.), Neurobiology and treatment of traumatic dissociation: Toward an embodied self (pp. 5-28). Springer Publishing Company. http: / / doi.org/10.1891/9780826106322

Laub, B., Nomi, W., \& Bender, S.S. (2017). A dialectical perspective on the adaptive information processing model and EMDR therapy. Journal of EMDR Practice and Research, 11(2), 111-119. https://doi. org/10.1891/1933-3196.11.2.111

Lee, C. W., Taylor, G., \& Drummond, P. D. (2006). The active ingredient in EMDR: Is it traditional exposure or dual focus of attention? Clinical Psychology \& Psychotherapy: An International Journal of Theory \& Practice, 13(2), 97-107. https: / / doi.org/10.1002/ cpp.479

Levine, P. A., \& Frederick, A. (1997). Waking the tiger: Healing trauma: The innate capacity to transform overwhelming experiences. North Atlantic Books.

Lewin, K. (1939). Field theory and experiment in social psychology: Concepts and methods. American Journal of Sociology, 44(6), 868-896. https://doi.org/ $10.1086 / 218177$

Liang, L. H., Brown, D. J., Lian, H., Hanig, S., Ferris, D. L., \& Keeping, L. M. (2018). Righting a wrong: Retaliation on a voodoo doll symbolizing an abusive supervisor restores justice. The Leadership Quarterly, 29(4), 443-456. https: / / doi.org/10.1016/j.leaqua.2018. 01.004

Liberman, N., Forster, J., \& Higgins, E. T. (2007). Completed vs. interrupted priming: Reduced accessibility from post-fulfillment inhibition. Journal of Experimental Social Psychology, 43, 258-264. https://doi.org/ 10.1016/j.jesp.2006.01.006

Mandler, G. (1964). The interruption of behavior. Proceedings of the Nebraska Symposium on Motivation, 12, 163-219.

Mantyla, T., \& Sgaramella, T. (1997). Interrupting intentions: Zeigarnik-like effects in prospective memory. Psychological Research, 60(3), 192-199. https: / / doi. org/10.1007/BF00419767
Mark, G., Gudith, D., \& Klocke, U. (2008). The cost of interrupted work: More speed and stress. Proceedings of the SIGCHI Conference on Human Factors in Computing Systems, Chi '08, 107-110. http://doi. org/10.1145/1357054.1357072

Mark, G., Voida, S., and Cardello, A. (2012). A pace not dictated by electrons: an empirical study of work without email. Proceedings of the SIGCHI Conference on Human Factors in Computing Systems, Chi '12, 555-564. http: / / doi.org/10.1145/2207676.2207754

Markus, W., \& Hornsveld, H. K. (2017). EMDR interventions in addiction. Journal of EMDR Practice and Research, 11(1), 3-29. https: / / doi.org/10.1891/1933-3196.11.1.3

Marrow, A. J. (1938). Goal tensions and recall: I. The Journal of General Psychology, 19(1), 3-35. https: / / doi.org/10.1080/00221309.1938.9711185

Martin, L. L., \& Tesser, A. (1989). Toward a motivational and structural theory of ruminative thought. In J. S. Uleman \& J. A. Bargh (Eds.), Unintended thought (p. 306-326). Guilford Press. https://doi. org/10.1002/mar.4220110109

Masicampo, E. J., \& Baumeister, R. F. (2011). Unfulfilled goals interfere with tasks that require executive functions. Journal of Experimental Social Psychology, 47, 300311. https: / / doi.org/10.1016/j.jesp.2010.10.011

McHugh, T., Forbes, D., Bates, G., Hopwood, M., \& Creamer, M. (2012). Anger in PTSD: Is there a need for a concept of PTSD-related posttraumatic anger? Clinical Psychology Review, 32(2), 93-104. https: / / doi.org/10.1016/j.cpr.2011.07.013

McNamara, J., \& Lister-Ford, C. (1995). Ego states and the psychology of memory. Transactional Analysis Journal, 25(2), 141-149. https://doi.org/10.1177/ 036215379502500207

Miller, P. W., McGowan, I. W., Bergmann, U., Farrell, D., \& McLaughlin, D. F. (2018). Stochastic resonance as a proposed neurobiological model for Eye Movement Desensitization and Reprocessing (EMDR) therapy. Medical Hypotheses, 121, 106-111. https: / / doi.org/10.1016/j.mehy.2018.09.010

Mioni, G., Santon, S., Stablum, F., \& Cornoldi, C. (2017). Time-based prospective memory difficulties in children with ADHD and the role of time perception and working memory. Child Neuropsychology, 23(5), 588608. https: / / doi.org/10.1080/09297049.2016.1172561

Paulsen, S., \& Lanius, U. (2009). Toward an embodied self; integrating EMDR with somatic and ego state interventions. In R. Shapiro (Ed.), EMDR solutions II: For depression, eating disorders, performance, and more (pp. 336-388). W.W. Norton \& Company, Inc.

Payne, P., Levine, P. A., \& Crane-Godreau, M. A. (2015). Somatic experiencing: Using interoception and proprioception as core elements of trauma therapy. Frontiers in Psychology, 6, 1-18. https://doi.org/ 10.3389/fpsyg.2015.00093

Peifer, C., Syrek, C., Ostwald, V., Schuh, E., \& Antoni, C. H. (2019). Thieves of Flow: How Unfinished Tasks 
at Work are Related to Flow Experience and Wellbeing. Journal of Happiness Studies, 21, 1641-1660. https: / / doi.org/10.1007/s10902-019-00149-Z

Pietrusza, C., \& Hook, D. (2016). 'You're kicking me out?' Scansion and the variable-length session in Lacanian clinical praxis. Psychodynamic Practice, 22(2), 102-119. https: / / doi.org/ 10.1080/14753634.2016.1162109

Popova, Y.B. (2015). Stories, meaning, and experience: Narrativity and enaction, 9. Routledge.

Reder, L. M. (1987). Strategy selection in question answering. Cognitive Psychology, 19(1), 90-138. https://doi. org / 10.1016/0010-0285(87)90005-3

Roşu, B. (2006). Tulburări psihice în expertiza medicolegală a leziunilor corporale. Romanian Journal of Legal Medicine, 14(1), 38-45.

Savitsky, K., Medvec, V. H., \& Gilovich, T. (1997). Remembering and regretting: The Zeigarnik effect and the cognitive availability of regrettable actions and inactions. Personality and Social Psychology Bulletin, 23(3), 248-257. https: / / doi.org/10.1177/0146167297233004

Schäflein, E., Sattel, H. C., Pollatos, O., \& Sack, M. (2018). Disconnected-impaired interoceptive accuracy and its association with self-perception and cardiac vagal tone in patients with dissociative disorder. Frontiers in Psychology, 9, 897. https://doi.org/ 10.3389/fpsyg.2018.00897

Schell, A. M., \& Catania, J. (1975). The relationship between cardiac activity and sensory acuity. Psychophysiology, 12(2), 147-151. https://doi.org/10.1111/j.14698986.1975.tb01265.x

Seifert, C. M., \& Patalano, A. L. (1991). Memory for incomplete tasks: A re-examination of the zeigarnik effect. In Preceedings of the thirteenth annual conference of the cognitive science society [refereed]. (pp. 114-119). Erlbaum.

Shapiro, F. (1995). Eye movement desensitization and reprocessing: Basic principles, protocols and procedures. Guilford Press.

Shapiro, F. (2001). Eye movement desensitization and reprocessing: Basic principles, protocols and procedures (2nd ed.). Guilford Press.

Shapiro, F. (2017). Eye movement desensitization and reprocessing (EMDR) therapy: Basic principles, protocols, and procedures (3rd ed.). Guilford Publications.

Sinclair, A. H., \& Barense, M. D. (2018). Surprise and destabilize: Prediction error influences episodic memory reconsolidation. Learning \& Memory, 25(8), 369-381. https: / / doi.org/10.1101/lm.046912.117

Sio, U. N., \& Ormerod, T. C. (2009). Does incubation enhance problem solving? A meta-analytic review. Psychological Bulletin, 135(1), 94. https://doi.org/ $10.1037 /$ a0014212

Smallwood, J., \& Schooler, J. W. (2006). The restless mind. Psychological Bulletin, 132(6), 946. https://doi. org/10.1037/0033-2909.132.6.946
Stocker, D., Keller, A. C., Meier, L. L., Elfering, A., Pfister, I. B., Jacobshagen, N., \& Semmer, N. K. (2019). Appreciation by supervisors buffers the impact of work interruptions on well-being longitudinally. International Journal of Stress Management, 26(4), 331-343. https: / / doi.org/10.1037/str000011

Smit, B. W. (2016). Successfully leaving work at work: The self-regulatory underpinnings of psychological detachment. Journal of Occupational and Organizational Psychology, 89(3), 493-514.

Syrek, C. J., Weigelt, O., Peifer, C., \& Antoni, C. H. (2017). Zeigarnik's sleepless nights: How unfinished tasks at the end of the week impair employee sleep on the weekend through rumination. Journal of Occupational Health Psychology, 22(2), 225.

Umeda, S., Tochizawa, S., Shibata, M., \& Terasawa, Y. (2016). Prospective memory mediated by interoceptive accuracy: A psychophysiological approach. Philosophical Transactions of the Royal Society B, 371(1708), 20160005. https: / / doi.org/10.1098/ rstb.2016.0005

Weigelt, O., \& Syrek, C. J. (2017). Ovsiankina’s great relief: How supplemental work during the weekend may contribute to recovery in the face of unfinished tasks. International Journal of Environmental Research and Public Health, 14(12), 1606. https:// doi.org/10.3390/ijerph14121606

Werner, N. S., Schweitzer, N., Meindl, T., Duschek, S., Kambeiz, J., \& Schandry, R. (2013). Interoceptive awareness moderates neural activity during decision-making. Biological Psychology, 94(3), 498-506. https: / / doi.org/10.1016/j.biopsycho.2013.09.002

West, R., Jakubek, K., \& Wymbs, N. (2002). Age-related declines in prospective memory: Behavioral and electrophysiological evidence. Neuroscience \& Biobehavioral Reviews, 26(7), 827-833. https:// doi. org/10.1016/S0149-7634(02)00069-6

Zeigarnik, B. (1927). Das Behalten erledigter und unerledigter Handlungen [The memory of completed and uncompleted actions]. Psychologische Forschung, 9, $1-85$.

Disclosure. The author has no relevant financial interest or affiliations with any commercial interests related to the subjects discussed within this article.

Funding. The author received no specific grant or financial support for the research, authorship, and/or publication of this article.

Correspondence regarding this article should be directed to Jeremy G. Fox, Atlanta, GA, USA. E-mail: foxemdrtherapy @gmail.com 